\title{
Immunohistochemical studies (ER \& Ki-67) in Proliferative breast lesions adjacent to malignancy
}

\author{
Dr. R. Sathyalakshmi ${ }^{1}$, Dr. A. Sundaram ${ }^{2}$, Dr. Chithra Srinivasan ${ }^{3}$, \\ Dr. Subachithra ${ }^{4}$ \\ ${ }^{I}$ (Department of Pathology, Stanley Medical College,The Tamilnadu Dr.MGR Medical University,India) \\ ${ }_{2}^{2}$ (Department of Pathology, SRM Medical College,SRM University,India) \\ ${ }_{3}^{3}$ (Department of Pathology, Saveetha Medical College,Saveetha University,India) \\ ${ }^{4}$ (Department of Pathology, Stanley Medical College,The Tamilnadu Dr.MGR Medical University,India)
}

\begin{abstract}
Breast carcinoma is the second most frequently occuring malignant tumor and the leading cause of cancer death in women. Breast carcinomas usually arise in a multistep fashion from intermediary lesions to invasive cancer. The presence of different lesions within the same breast adjacent to malignancy is a phenomenon that can prove the multistep progression to malignancy $(1,3)$. The aim of this study was to identify the predominantly occurring lesion adjacent to malignancy and study the histological appearance, Proliferative Ki-67 index (MIB-1 index) and Estrogen receptor (ER) status in such lesions and substantiate their possible identity as a premalignant lesion in that particular case. Adjacent lesions were categorized as non proliferative lesions, proliferative lesions with and without atypia and DCIS. It was observed that the predominant lesions occurring adjacent to malignancy were proliferative lesions with and without atypia. ER and Ki-67 values were high in those cases with adjacent lesions with atypia. The high positivity of ER and Ki 67 in the adjacent proliferative lesions with atypia hence proves that these could have been the precursors which had progressed to malignancy in these cases.

Hence finding of lesions in benign breast biopsies showing a high proliferative rate and ER positivity necessitates regular follow up of these patients and helps in identifying early progression of these lesions to malignancy.Initiating therapy to these patients at an earlier stage can reduce the incidence of breast cancer.
\end{abstract}

Key Words: Breast carcinoma, proliferative breast lesions adjacent to malignancy, Estrogen Receptor status , Proliferative index

\section{Introduction}

Breast carcinoma is the second most commonly occurring solid epithelial tumors in women next to cancer cervix. In our Department of Pathology, Stanley Medical College we received a total of 18,968 cases for histopathological examination during Jan 2006 to Sep 2008 and 694 of those cases were breast lesions. Benign breast lesions constituted about 482 cases and about 212 cases were malignant.

Breast carcinomas usually arise in a multistep fashion from intermediary lesions to invasive cancer $(1,3)$. The identification of premalignant lesions hence play a very important role in preventing progression to malignancy.

The presence of different lesions within the same breast adjacent to malignancy is a usual phenomenon proving the multistep progression to malignancy $(1,3)$. Hence identifying such predominantly occurring lesions adjacent to malignancy and studying of Ki-67 proliferative index (MIB-1 index) and Estrogen receptor (ER) status in such lesions could substantiate their possible identity as premalignant lesion in that particular case.

This work aims at histologically identifying such high risk lesions occurring adjacent to malignancy and confirming their risk status by MIB 1 index and ER status.The association between ER positivity and Ki 67 index has also been studied in this work to establish that they correlate proportionately. The study tries to establish the lesions which have the potential for progression to overt malignancy, thereby indicating early identification and appropriate treatment to reduce the incidence of breast malignancies.

\section{Materials And Methods}

The total breast specimens received during the study period of two and a half years from 2006 July to 2008 December was 694, out of which benign lesions were 482 and malignant lesions constituted 212. 50 cases of Radical and simple mastectomy proved histologically malignant were selected at random for this sudy. Sampling was done from all four quadrants surrounding the malignant lesion and the tissues were subjected to routine processing and stained with Hematoxylin \& Eosin. Slides from the nearest point where tumor infiltration was not seen were studied and the lesions were classified morphologically (Table: 1).

Based on the predominant lesions present the lesions were classified as 
mild $-<25 \%$,

moderate $-26-50 \%$, and

florid - >50\%

20 cases representing lesions from all the above categories were selected from the total of 50 cases for study of ER and Ki-67 status.

Ki67

MIB 1 labelling indices were estimated as a percentage of positively stained nuclei of atleast 100 cells counted in three or more fields of a given lesion or foci at a magnification of $400 \mathrm{x}$. The cut off value to define High Ki 67 proliferation index was set at $30 \%$ (3).

RESULTS

- Category A - Benign non proliferative lesions - 19 cases

- Category B - Proliferative lesions without atypia - 22 cases

- Category $\mathrm{C}$ - Proliferative lesions with atypia and in situ changes -9 cases

Most frequently(about 60\%) occuring lesions adjacent to malignancy was usual ductal hyperplasia (Table 3)

\section{Ihe Results}

A total of 20 cases were selected for immunohistochemical studies -5 cases which showed non-proliferative lesions , 8 cases which showed proliferative lesions without atypia and 7 cases which showed atypia .

\section{ER VALUES}

ER positivity was found in 9 cases out of 20. Proliferative lesions with atypia including in situ lesion showed increased ER(Fig 4) and majority of the proliferative lesions without atypia showed ER negativity and few showed ER positivity(Fig 3).

\section{Ki-67 VALUES}

Ki-67 positivity was found in 7 cases of proliferative lesions with atypia and 8 cases of proliferative lesions without atypia(Fig 5).The proliferative index values are very high in case of lesions belonging to Atypical ductal hyperplasia( Fig 6) and DCIS.

\section{Discussion}

The incidence and mortality due to breast malignancy are high in women. Breast carcinomas arise in a multistep fashion through a series of intermediate lesions to invasive cancer and hence the identification of premalignant lesions involved in the development of breast cancer becomes very essential.

An attempt was made here to ascertain the association between these adjacent changes and the malignant lesion. This study also tried to substantiate that these changes could be the probable premalignant lesion in these cases. The adjacent changes were studied and placed under three categories based on the predominant lesion seen by grading the lesions as mild, moderate and florid.

Category A - Benign non proliferative lesions

Category B - Proliferative lesions without atypia(Fig 1)

Category $\mathrm{C}$ - Proliferative lesions with atypia(Fig2) and in situ changes.

On analyzing the results it was found that the lesion occurring predominantly adjacent to malignancy was Usual Ductal Hyperplasia.

Proliferative index and ER status were also studied in these cases These studies have recently received increased attention as increased proliferative index signifies the lesion is a fertile soil for future malignancy. Estrogens are mitogenic to breast epithelial cells and the resulting proliferation increases the probability of mistakes made in the DNA replication and setting these mistakes as mutations.

In the current study of Ki 67 positivity was found to be high in categories B and Andreas Ludwig and Bettina et al has showed similar results in their study (Table 5 )

Previous literature (4) also cites that high proliferative index in adjacent area suggest an invasive carcinoma in the neighbourhood and hence the results of High Ki 67 in the adjacent lesions suggest that it could have been the premalignant lesion for the current malignancy.

Normal premenopausal terminal duct lobular units contain about $30 \%$ of ER positive cells and nearly all cells express very high ER levels in the majority of premalignant breast lesions( 5). The results obtained from this study also correlates with the literature stating the high positivity of ER in atypical lesions. Harold J. Burnstein in his study (Table 6) has also showed that ER positivity in DCIS is low in high grade lesions.

The results of this study hence correlates with the literature that the ER negative DCIS are high grade and this was also showed histologically (comedo and solid types of DCIS). Previous studies (6) state that ER positivity and $\mathrm{Ki} 67$ index are high in cases of premalignant lesions of the breast and this fact is proved in our 
study. On comparing the cases of ER positivity and High Ki 67 index it was observed that both were directly proportional.

In conclusion this study showed that the predominant lesion found adjacent to malignancy was usual ductal hyperplasia.Ki-67 index were high in adjacent areas showing proliferative lesions with and without atypia. ER values were high in proliferative lesions with atypia and in situ leisons. ER positivity and Ki-67 rate showed a direct proportional increase. This high positivity of ER and Ki-67 in the adjacent proliferative lesions with atypia hence proves that these could have been the precursors which have progressed to malignancy in these cases. Hence finding of these proliferative lesions in benign breast biopsies showing a high proliferative rate and ER positivity necessitates regular follow up of these patients and helps in identifying early progression of these lesions to malignancy and initiating treatment to these patients at an earlier stage and thereby reduce the incidence of breast cancer.

\section{V. $\quad$ Er And Ki-67 Comparison}

This study has shown that proliferative lesions with atypia and some DCIS cases which showed ER positivity also showed a high Ki-67 index. Anderson et al in his publication has also stated that the proportion of ER positive / Ki-67 positive proliferating cells is increased in all risk associated lesions of the breast and it increases from normal breast lobules through hyperplasia to insitu cancer and invasive cancer

\section{Ki-67 Proliferation Index}

In normal breast $\mathrm{Ki}-67$ is expressed at a very low level ( $<3 \%$ of cells ). In our study 7 cases of proliferative lesions with atypia and 8 cases of proliferative lesions without atypia showed high proliferative index, 4 out of 5 cases in the benign non-proliferative lesion category showed low proliferation rate and one case showed high proliferative index. This high value is probably due to the UDH found focally in this case. High proliferative index in adjacent area suggest an invasive carcinoma in the neighbourhood and hence the results of High Ki 67 in the adjacent lesions suggest that it could be the premalignant lesion for the current malignancy.They could have been the fertile soil leading on to malignancy in these cases (4)

\section{Association Between Er And Ki-67}

In normal breast tissue ER and the proliferation associated antigen Ki-67 are negatively associated. ER positive cells are non dividing or the receptor is down regulated as cells enter the cycle.This relation is completely or partially lost in many ER positive breast cancer and in in- situ proliferations associated with an increased cancer risk, where co-expression of the two markers is also found.

\section{References}

[1] Hartmann L C, Sellers TA, Frost MH, Lingle WL, Degnim AC, Ghosh K, Vierkant RA, Maloney SD, Pankratz VS, Hillman DW et al: Benign breast disease and risk of breast cancer - New England Journal of Medicine Vol - 353:229-237 July 2005.

[2] Dupont W D, Page D L - Risk factors for breast cancer in women with proliferative breast disease - New England J of Medicine 1985,312:146-151.

[3] Ki 67 - a luminal marker that identifies high risk subgroup in hormone receptor positive and node negative breast cancer - Journal of Clinical Oncology June 2007 ASCO Annual Meeting Proceeding Part 1 Volume 25.

[4] Fuelgen DNA content and mitotic activity in proliferative breast disease - A comparison with DCIS - Biomarkers of Proliferative breast disease - 1995 The Breast Journal 1:4,222-226

[5] Shoker B S et al - Estrogen receptor expression in normal and precancerous breast - J pathology 1999 July,188(3):237-244

[6] Anderson E, Munro C, Davies M PA, Sibson D R, Sloane J P - Abnormal regulation of Estrogen receptor in benign breast disease Journal of Clinical Pathology, Oct 2000,53(10):778-783.

[7] Grazia Arpino, Laucirica R, Elledge RM : Premalignant and Insitu Breast Disease Biology and clinical implications - Annuals of Internal Medicine Sep 20, 2005 - Vol 143.Iss 6. pg 446-457.

[8] Barnes D M, Millis RR - 1995 Oestrogen receptors - the history, the relevance and methods of evaluation

TABLE: 1

\begin{tabular}{|l|l|}
\hline Category A & Cyst formation \\
\cline { 2 - 2 } Non proliferative(fibrocystic) changes & Apocrine changes \\
\cline { 2 - 2 } & Hyalinization and calcification \\
\cline { 2 - 2 } & Mild epithelial hyperplasia \\
\hline \multirow{5}{*}{$\begin{array}{l}\text { Category B } \\
\text { Proliferative lesions without atypia }\end{array}$} & Fibroadenoma \\
\cline { 2 - 2 } & Intraductal papilloma and papillomatosis \\
\cline { 2 - 2 } & Microglandular adenosis \\
\cline { 2 - 2 } & Blunt duct adenosis \\
\cline { 2 - 2 } & Radial scar/complex sclerosing lesions \\
\cline { 2 - 2 } & Sclerosing adenosis \\
\cline { 2 - 2 } & Moderate and florid hyperplasia \\
\hline Category C & Atypical ductal hyperplasia \\
\cline { 2 - 2 } Proliferative lesions with atypia & Atypical lobular hyperplasia \\
\cline { 2 - 2 } In situ lesions & Ductal carcinoma in situ \\
\cline { 2 - 2 } & Lobular carcinoma in situ \\
\hline
\end{tabular}


TABLE 2 ER ASSAY METHOD Quick score (Barnes et al 1995)

- Assigning value to both intensity and proportion of positivity .

$\underline{\text { Score for intensity }}$

Score for proportion of positivity

\begin{tabular}{|l|l|}
\hline no staining & $\underline{0}$ \\
\hline weak staining & $\underline{1}$ \\
\hline moderate staining & $\underline{2}$ \\
\hline strong staining & $\underline{3}$ \\
\hline
\end{tabular}

\begin{tabular}{|l|l|}
\hline no staining & $\underline{0}$ \\
\hline$<1 \%$ of nuclei staining & $\underline{1}$ \\
\hline $1 \%-10 \%$ of nuclei staining & $\underline{2}$ \\
\hline $11 \%-33 \%$ of nuclei staining & $\underline{3}$ \\
\hline $34 \%-66 \%$ of nuclei staining & $\underline{4}$ \\
\hline $67 \%-100 \%$ of nuclei staining & $\underline{5}$ \\
\hline
\end{tabular}

The scores were summed to give a maximum of 8 .

Scores 2 or less were regarded as ER negative

TABLE 3 Proliferative Breast Lesions

\begin{tabular}{|l|l|l|l|l|}
\hline Proliferative breast lesions & Mild & Moderate & Florid & Total \\
\hline Usual ductal hyperplasia & 15 & 8 & 7 & 30 \\
\hline Atypical ductal hyperplasia & 5 & 3 & 1 & 9 \\
\hline In situ lesions & 9 & 1 & 1 & 11 \\
\hline
\end{tabular}

TABLE 4

\begin{tabular}{|l|l|l|}
\hline Type of lesion & No of cases & Proliferative index \\
\hline Proliferative lesions with atypia & 7 & High \\
\hline Proliferative lesions without atypia & 8 & High \\
\hline Benign non-proliferative lesions & $4 / 5$ & High \\
\hline
\end{tabular}

TABLE No : 5

\begin{tabular}{|l|l|l|l|}
\hline Diagnosis & $\begin{array}{l}\text { Mean(\%) } \\
(\mathbf{m i n}-\mathbf{m a x})\end{array}$ & $\begin{array}{l}\text { Median } \\
(\%)\end{array}$ & $\begin{array}{l}\text { Standard } \\
\text { deviation(\%) }\end{array}$ \\
\hline Normal breast & $1.83(0-11.93)$ & 0.99 & $+/-2.644$ \\
\hline Fibrocystic lesion & $0.37(0-4.76)$ & 0 & $+/-0.99$ \\
\hline Adenosis & $3.23(0-17.60)$ & 1.96 & $+/-3.39$ \\
\hline UDH & $2.41(0-20.64)$ & 0.99 & $+/-4.02$ \\
\hline DCIS (non high grade) & $8.40(0-20.00)$ & 7.83 & $+/-5.32$ \\
\hline
\end{tabular}

TABLE : 6

\begin{tabular}{|l|l|l|l|}
\hline \multirow{2}{*}{ ER status } & Tumor grade & 3 \\
\cline { 2 - 4 } & 1 & 2 & $61 \%$ \\
\hline Positive & $87 \%$ & $85 \%$ & $39 \%$ \\
\hline Negative & $13 \%$ & $15 \%$ & 6 \\
\hline
\end{tabular}

PICTURES

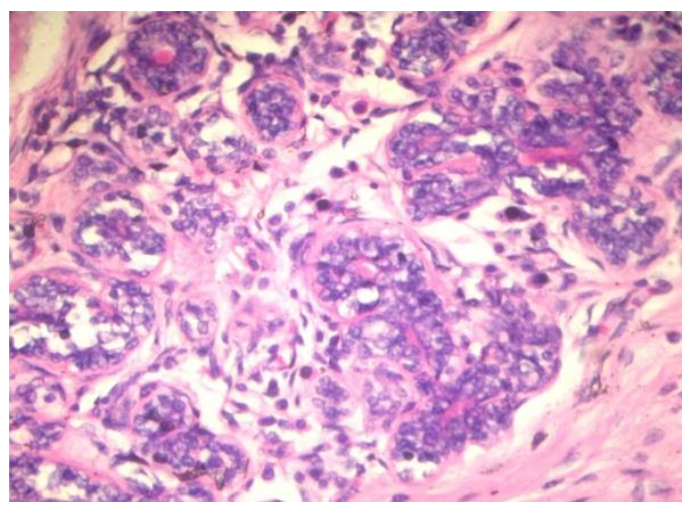

Figure 1: Moderate epitheliosis 


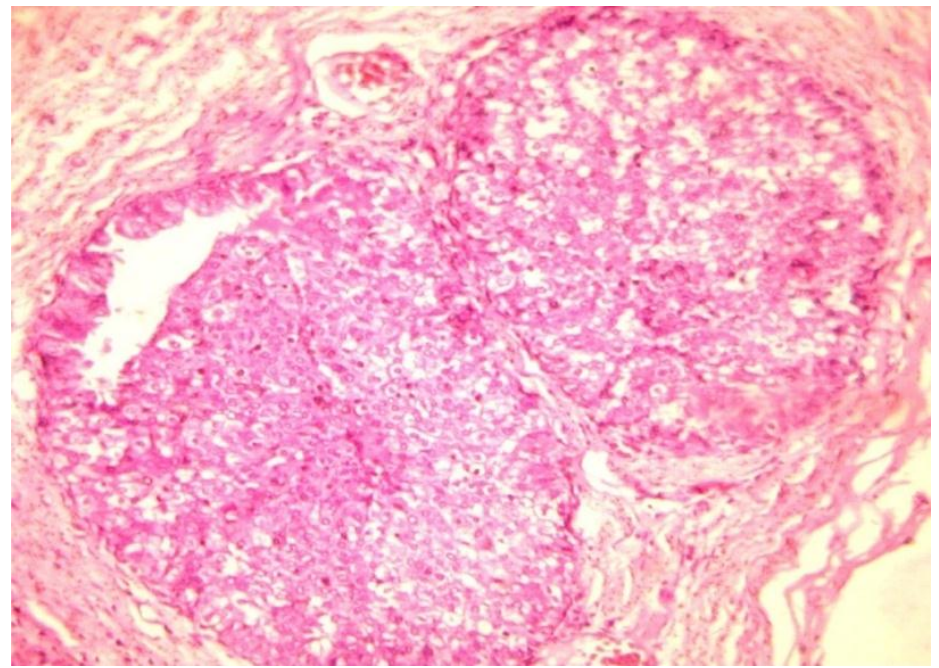

Figure 2: Atypical ductal hyperplasia

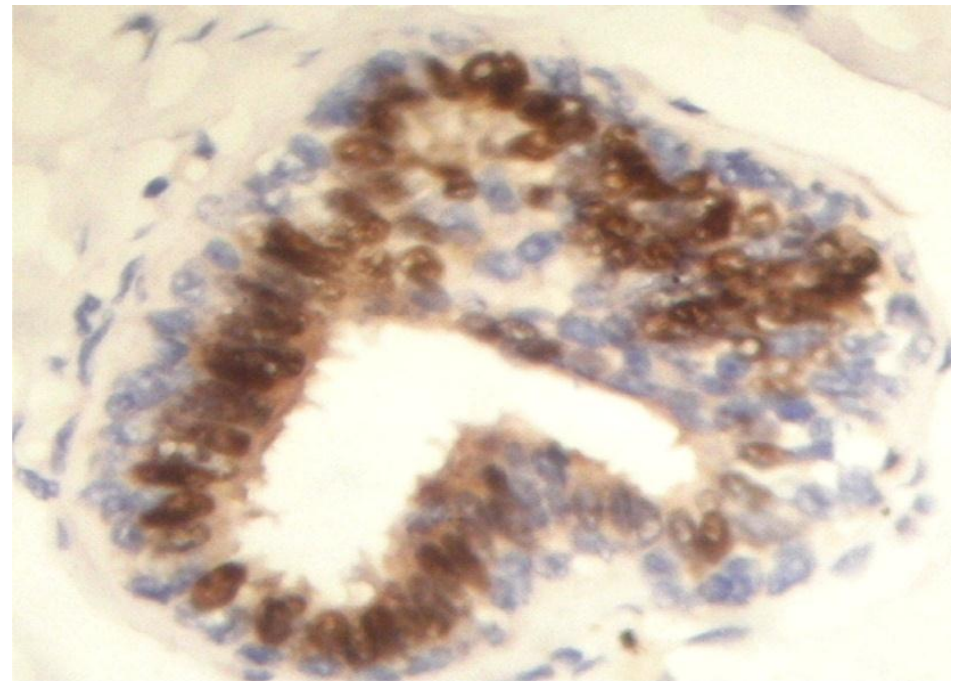

Figure 3: ER positivity in Moderate epitheliosis

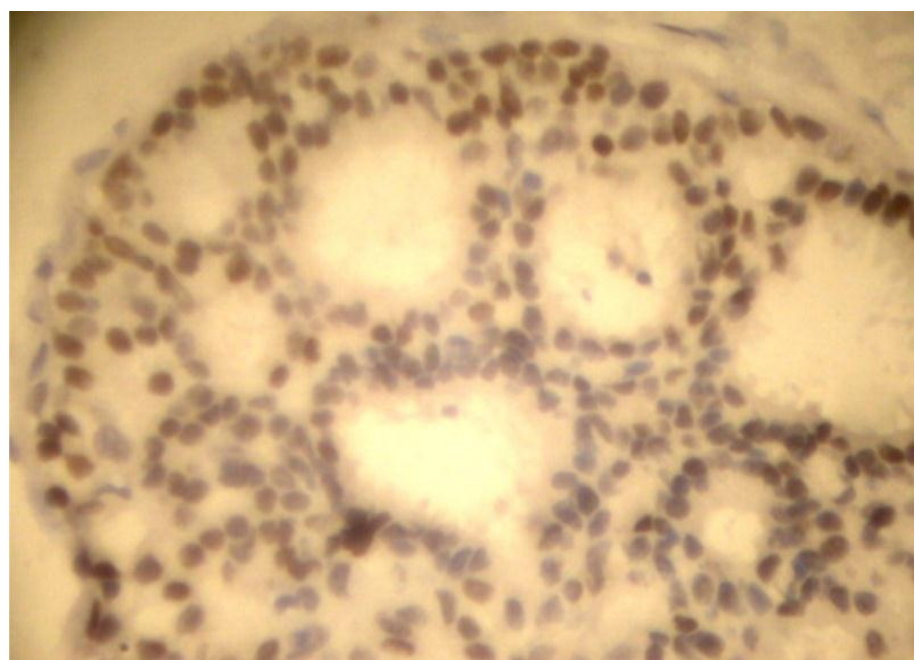

Figure 4: ER positivity in ADH 


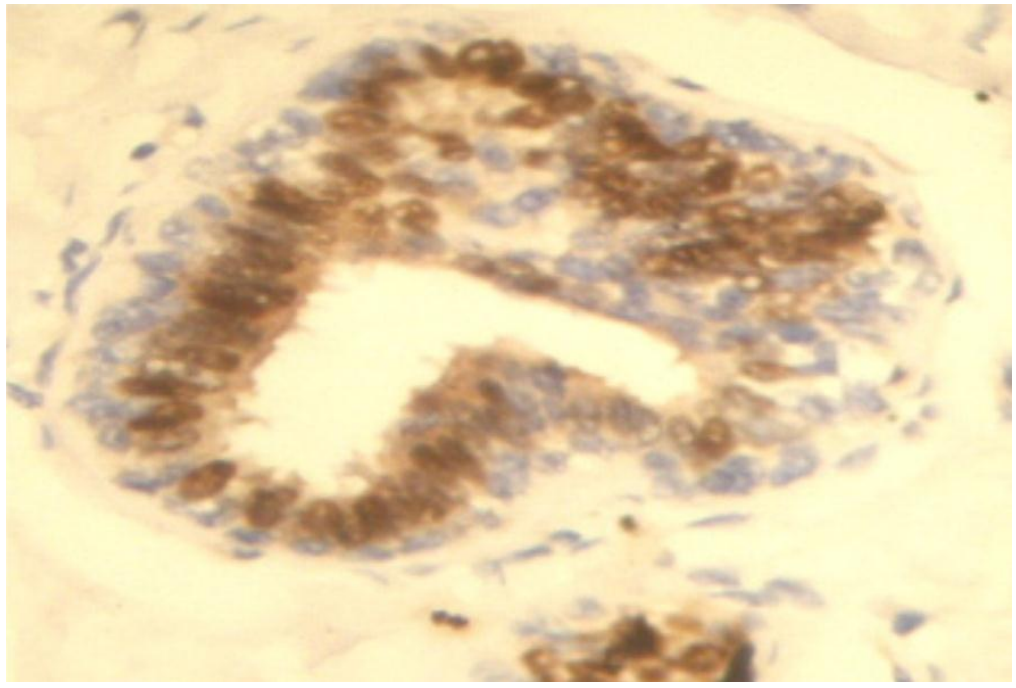

Figure 5: Ki 67 positivity in moderate epitheliosis

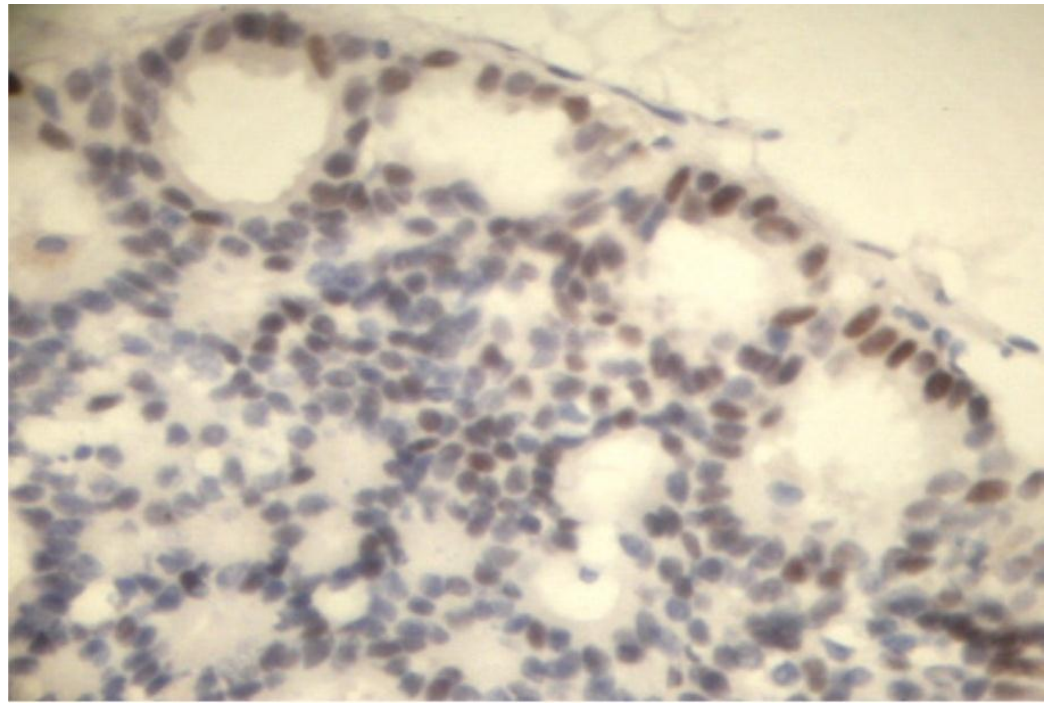

Figure 6: Ki 67 positivity in ADH 